\title{
Evolución de la revelación de información no financiera: evidencia desde el contexto colombiano*
}

\author{
Evolution of the disclosure of non-financial information: evidence from the Colombian context \\ Evolução da divulgação de informação não financeira: evidência do contexto colombiano
}

\author{
Juan David Villa-Barrientos \\ Universidad de Antioquia, Colombia \\ ORCID: https://orcid.org/0000-0001-7061-4671 \\ Yuliana Escobar-Cruz \\ Universidad de Antioquia, Colombia \\ ORCID: https://orcid.org/0000-0001-7774-484X \\ Diego Andrés Correa-Mejía ${ }^{\mathrm{a}}$ \\ Universidad de Antioquia, Colombia \\ diegoa.correa@udea.edu.co \\ ORCID: https://orcid.org/0000-0002-1319-0451
}

DOI: https://doi.org/10.11144/Javeriana.cc21.erif

Recibido: 17 Marzo 2020

Aceptado: 24 Julio 2020

Publicado: 18 Diciembre 2020

\section{Resumen:}

El objetivo de este artículo es analizar la evolución en las revelaciones no financieras de los reportes corporativos en Colombia. La muestra seleccionada incluye 19 empresas pertenecientes al índice COLCAP entre los años 2013-2018, utilizando como unidad de análisis los reportes corporativos, agrupados en cuatro categorías: Reporte Anual (RA), Reporte Combinado (RC), Reporte de Sostenibilidad (RS) y Reporte Integrado (RI). Los resultados obtenidos se dividen en tres grupos: en una primera parte se encuentra que los informes integrados presentan la mayor cantidad de publicaciones, y las empresas con mayores revelaciones se localizan en el sector industrial e inversión. En cuanto al comportamiento de las revelaciones en materia ambiental, social, económico, gobierno corporativo, se obtuvo mayor frecuencia de divulgaciones relacionadas con el gobierno corporativo y temas sociales, seguidos de información revelada sobre asuntos medio ambientales y temas económicos. Por último, en cuanto al compromiso con los objetivos de desarrollo sostenible se halló que los objetivos relacionados con el trabajo decente y educación de calidad tienen intervención importante en el contenido de los reportes corporativos.

Código JEL: G30

Palabras clave: Reporte corporativo, responsabilidad social empresarial, gobierno corporativo, objetivos de desarrollo sostenible.

\begin{abstract}
:
The objective of this article is to evaluate the evolution in corporate reports in Colombia. The selected list included 19 companies belonging to COLCAP index between 2013-2018, using corporate reports as a unit of analysis, grouped into four categories: Annual report (AR), Combined reports (CR), Sustainability reports (SR) and Integrated reports (IR). The results obtained are divided in three groups: In the first part it is found that the integrated reports present the greatest amount of publications, and the companies with the greatest disclosures are located in the industrial and investment sector. As the behavior of disclosures in environmental, social, economic and corporate governance, greater frequency of disclosures related to corporate governance and social issues was obtained, followed by revealed information on environmental issues and economic issues. Finally, the commitment with sustainable development goals, it was found that the goals related to decent work and quality education have important intervention in the content of corporate reports.
\end{abstract}

JEL Code: G30

Keywords: Corporate reports, corporate social responsibility, corporate governance, sustainable development goals.

\section{Resumo:}

O objetivo deste artigo é avaliar a evolução nas divulgações dos relatórios corporativos na Colômbia. A amostra selecionada inclui 19 empresas pertencentes ao índice COLCAP entre 2013-2018, utilizando os relatórios corporativos como unidade de análise, agrupados em quatro categorias: Relatório Anual (RA), Relatório Combinado (RC), Relatório de Sustentabilidade (RS)

Notas de autor

a Autor de correspondencia. Correo electrónico: diegoa.correa@udea.edu.co 
e Relatório Integrado (RI). Os resultados obtidos são divididos em três grupos: na primeira parte, verificase que os relatórios integrados apresentam a maior quantidade de publicações, e as empresas com as maiores divulgações estão localizadas no setor industrial e de investimentos. Quanto ao comportamento das divulgações em questões ambientais, sociais, econômicas, de Governança Corporativa, obteve-se maior frequência de divulgações relacionadas à governança corporativa e questões sociais, seguidas de informações reveladas sobre questões ambientais e econômicas. Por fim, constatou-se o comprometimento com os objetivos do desenvolvimento sustentável que os objetivos relacionados ao trabalho decente e à educação de qualidade têm importante intervenção no conteúdo dos relatórios corporativos.

Código JEL: G30

Palavras-chave: Relatório corporativo, responsabilidade social corporativa, governança corporativa, objetivos de desenvolvimento sustentável.

\section{Introducción}

Los reportes corporativos se componen tanto de la información financiera, como de información no financiera a través de reportes de sostenibilidad, integrados o combinados, y son un mecanismo de comunicación entre las empresas y los grupos de interés que facilita el crecimiento económico empresarial (Price \& Sun, 2017). La comunicación de los avances en temas sociales, medioambientales, económicos y gobierno corporativo es un tema de investigación actual (Gürlek, Düzgün, \& Uygur, 2017; Gürlek \& Tuna, 2019; Kansal, Joshi, \& Batra, 2014). Dado que los reportes corporativos brindan información de carácter no financiero, las organizaciones que los han implementado han encontrado una estrategia efectiva para influenciar a sus Stakeholders (Austin \& Gaither, 2017; Sánchez, Rivera, \& Jaque, 2014).

Si bien la investigación en la comunicación de la Responsabilidad Social Empresarial -RSE- ha sido explorada, no se tienen muchos registros sobre los indicadores sociales, económicos, medioambientales y de gobierno corporativo revelados por las organizaciones relevantes en las dinámicas económicas locales. La investigación se centra en las empresas con mayor capitalización bursátil pertenecientes a la bolsa de valores de Colombia -BVC-, un país que para el 2018 contaba un crecimiento del producto interno bruto -PIB - mayor al de América latina (Superintendencia de Sociedades, 2019) y con historial en las prácticas de Responsabilidad Social Empresarial (Acosta \& Pérezts, 2019).

El objetivo de este artículo es analizar la evolución en las revelaciones de los reportes corporativos de compañías listadas en la bolsa de valores de Colombia. Para lograr este propósito se aplica un análisis de contenido de 19 empresas incluidas en el COLCAP, considerando sus revelaciones durante los años 2013-2018, llegando a una muestra final de 110 reportes agrupados y procesados a través de un análisis matricial.

De acuerdo con Dávila, Rodríguez y Elvira (2018), para la agrupación de los reportes se tienen cuatro categorías: Reporte Anual (RA), Reporte Combinado (RC), Reporte de Sostenibilidad (RS) y Reporte Integrado (RI). Y con fundamento en la teoría de los stakeholders (Freeman \& McVea, 1984), la teoría de la institución (DiMaggio \& Powell, 1983), teoría de la legitimidad (Ashforth, 2014; Guthrie \& Parker, 1989) y la teoría del desarrollo sostenible (Sharachchandra, 1991), en el desarrollo del artículo se definen aspectos tales como las partes interesadas, el tipo de información, mensaje a trasmitir (Robinson \& Eilert, 2018), y las formas de interacción entre las empresas y sus agentes de interés.

Algunos de los resultados más importantes se relacionan con el crecimiento de revelaciones relacionadas con información en materia social, aunque el tema con mayor revelación está inmerso en lo ambiental. Se observa un compromiso de las organizaciones con la calidad del aire y el calentamiento global como pilares fundamentales en el desarrollo y creación de valor a largo plazo.

El presente artículo se estructura de la siguiente manera: en la primera sección se presenta un contexto teórico, en la segunda sección explica la metodología, luego se exponen los resultados del estudio, y finalmente se presentan las conclusiones, así como también la sugerencia de futuras líneas de investigación. 


\section{Revisión de la literatura}

El reporte corporativo es el medio de comunicación entre las organizaciones empresariales y la sociedad, donde se le informa a las partes interesadas sobre los esfuerzos de las empresas para mejorar el equilibrio de sus aspectos económicos, ambientales y sociales (Freundlieb \& Teuteberg, 2013). Existen grandes ventajas competitivas de las entidades que utilizan los reportes corporativos como forma de comunicación, los cuales están siendo impulsados por una creciente presión legislativa en las empresas para proporcionar información sobre sus esfuerzos de sostenibilidad, pero sobre todo para legitimar sus acciones (Cuevas, Maldonado, \& Escobar, 2013; Sánchez et al., 2014).

Diferentes teorías respaldan lo dicho anteriormente, entre ellas la teoría de la legitimidad, en la cual se postula que las revelaciones corporativas son hechas como reacciones a factores ambientales, económicos, políticos y sociales, buscando legitimar las acciones organizacionales (Preston \& Post, 1981; Hogner, 1982; Lehman, 1983; Lindblom, 1983). Esta teoría es ampliamente reactiva, pues sugiere que los objetivos de la organización están estrechamente relacionados con los valores sociales implicados en sus actividades y en las normas sociales (Lindblom, 1983).

Por otro lado, la teoría de los Stakeholders, la cual define los agentes de interés como aquellos grupos sin cuyo apoyo la organización dejaría de existir, como los accionistas, empleados, clientes y prestamistas (Fernández \& Bajo, 2012). Esta definición ha tenido cambios, actualmente se dice que los Stakeholders son aquellos grupos que pueden afectar o son afectados por el logro de los objetivos de la organización, por lo cual incluye además a partes como el gobierno, medios de comunicación, sociedad y otros (Retolaza, San José, \& Ruiz, 2010). Este enfoque de los Stakeholders puede enriquecer la forma en que se gestionan las organizaciones, debido al nuevo concepto de empresa, la cual debe de tener en cuenta para la gestión de la misma a todos los grupos de interés que se relacionen con la organización (Freeman, 1984).

Estas teorías dan sustento teórico para el proceso de toma de decisiones, llevado a cabo por los Stakeholders, lo que lleva a resaltar la importancia en la forma como evoluciona la revelación de información no financiera para su análisis (Eccles \& Saltzman, 2010). Para entender la evolución del reporte corporativo es necesario considerar la existencia de dos fases en la literatura: la primera tiene que ver con las iniciativas de informes no financieros en la era de la sostenibilidad y la siguiente es la revolución de la información integrada (Dragu $\&$ Tiron, 2013a).

Los reportes corporativos siempre se han identificado por el contenido de información financiera, pero la información no financiera nace con los informes de sostenibilidad que tienen su origen en el concepto de RSE (González, 2007). Los informes de sostenibilidad se hicieron más visibles con el derrame de petróleo de Exxon Valdez en 1989 (Rupley, Brown, \& Marshall, 2017) en donde diferentes grupos se dieron a la tarea de divulgar los riesgos ambientales que conllevaban las operaciones de las organizaciones, lo que motivó a que las empresas empezaran a informar sobre hechos de sostenibilidad y, por ende, el número de reportes de información no financiera consiguiera aumentar en los años siguientes (Dragu \& Tiron, 2013a). Es por ello que inversores con mentalidad social crearon la Coalition for Environmentally Responsible Economies (CERES) (Rupley et al., 2017), y en 1997 con el fin de estandarizar los informes que contuvieran información de dicha índole, la CERES creó el Global Reporting Initiative (GRI) (Radley, 2012).

Para 1999 la organización AccountAbility (AA) desarrolló la norma AA1000, para la emisión de información no financiera, soporte para muchos de los informes de gestión conocidos en la actualidad (Maroun, 2017). En este sentido, estándares como el GRI, los principios de la organización para la cooperación y el desarrollo económico -OCDE-, las normas AA1000, SA8000 y la ISO 14001, son una extensión de las iniciativas que, en concordancia con los objetivos del Pacto Global, pretenden garantizar que el desarrollo esté ligado a la sostenibilidad (Medina \& Alvarez, 2011).

Por otra parte, las primeras publicaciones sobre informes integrados se presentaron en 2002, donde algunas compañías hicieron este reporte, como es el caso de Natura (Brasil) y Novozymes (Dinamarca) (Rivera, Zorio, 
\& García, 2016) en un período donde los acontecimientos mundiales estaban cambiando la perspectiva de la información no financiera, y por lo tanto era necesario un marco común para el desarrollo futuro de la información integrada. Como resultado se originó una tendencia de informes integrados, motivo por el cual en 2010 surge el International Integrated Reporting Committee, posteriormente denominado International Integrated Reporting Council (IIRC), que en 2011, emitió su primer borrador sobre informes integrados, abarcando los elementos de contenido y los principios para su elaboración (Dragu \& Tiron, 2013b; Sáez Martín, Haro de Rosario, \& Caba Pérez, 2014) y en 2013 emitió el marco Internacional de Reportes Integrados (IR), siendo esta una guía de aplicación voluntaria para la elaboración de Reportes Integrados (Rivera et al., 2016).

Las nuevas corrientes de los reportes corporativos indican que las compañías están adoptando prácticas con el fin de proveer información, la cual suministre mayor confianza a los Stakeholders, como por ejemplo el aseguramiento de la información de índole no financiero (Sìmnett, Vanstraelen, \& Chua, 2009). Ballou et al. (2018), aseguran que más del 66\% de las 250 mayores compañías del mundo realizan esta práctica, lo cual es un indicio de que las empresas han pasado de buscar solamente utilidades, para buscar una gestión sostenible (Schönborn et al., 2019) que les ayude además a enfrentar situaciones de crisis o fraude como el caso de Enron y WorldCom.

Los objetivos de desarrollo sostenible -ODS- también hacen parte de las nuevas tendencias de los reportes corporativos. Estas prácticas de revelaciones fortalecen la conciencia en el uso de los recursos para el progreso de la sociedad, y al igual que el aseguramiento su avance se efectuó a partir de la crisis económica global (Duran et al., 2015). La primera visión del concepto de desarrollo sostenible data de 1951 en donde la unión internacional para conservar la naturaleza publicó un informe sobre el medio ambiente global, posteriormente en 1972 se llevó a cabo la primera conferencia de la ONU relacionada a cuestiones ambientales, la convención de Viena en 1985, el reporte Brundtland en 1987, el protocolo de Kioto en 1997, el tratado de Ámsterdam en 1999, la Declaración del Milenio de 2000, la Cumbre de la tierra de Río en 2012. Los objetivos de desarrollo sostenible desarrollados por las Naciones Unidas, en 2015, abarcan los tres pilares fundamentales de la responsabilidad social empresarial, dimensión ambiental, económica y social, desarrollados en 17 objetivos, de los cuales cuatro corresponden a la dimensión ambiental, ocho a la social, cuatro a la económica y uno es transversal.

Los trabajos de aseguramiento y las nuevas tendencias en los reportes corporativos de la organización han develado la necesidad de información integrada que los informes de sostenibilidad y financieros no han logrado proporcionar (Pavlopoulos, Magnis, \& Iatridis, 2019). Diferentes estudios indican que los IR mejoran la reputación de la organización (Cortesi \& Vena, 2019; Pavlopoulos et al., 2019). Además, según el IIRC, los reportes integrados ayudan en la toma de decisiones a largo plazo, beneficiando así las estrategias futuras de las corporaciones e inversionistas, a diferencia de los diferentes informes de gestión, ya que estos no proporcionan información suficiente en materia de sostenibilidad organizacional (Havlová, 2015; Pavlopoulos et al., 2019). Los primeros informes de reporte integrado se llevaron a cabo con el plan piloto del IIRC entre 2011 y 2014, en donde participaron, en su mayoría, corporaciones europeas y la República de Sudáfrica (Havlová, 2015; Rivera et al., 2016). A partir de 2014 los informes integrados se conciben como la evolución de los reportes corporativos, tienen por objetivo mostrar cómo las organizaciones generan valor, luego no es simplemente un sumario entre la información financiera y no financiera sino más bien una red que las conecta (Maroun, 2017; Sáez Martín et al., 2014).

El IR se ha promovido como la solución a la cuestión del aislamiento en la información, sin embargo hay que tener en cuenta que las necesidades de información cambian, por lo que se requiere que los reportes corporativos estén al día con la demanda de información que los grupos de interés necesitan para la toma de decisiones de manera combinada dado que este tipo de información presenta un creciente apogeo (Rupley et al., 2017), requiriéndose reportes que den cuenta e integren aspectos financieros, sociales y medioambientales (Stewart, 2015). 
La presentación de informes bajo el concepto de integrados, ha sido reconocida solo desde 2010, como la mejor manera de obtener una visión completa del valor de las empresas, superando las limitaciones de los informes tradicionales (Eccles \& Krzus, 2010; Jensen y Berg, 2012; Abeysekera, 2013). Entre estos están, el Reporte anual (AR) que incluye información financiera y una sección dedicada a temas de sostenibilidad; el reporte de sostenibilidad (SR) que es un informe independiente del financiero; el reporte combinado (CR) el cual incluye los estados financieros consolidados y las notas a los estados financieros y el informe de sostenibilidad (RS) en un solo informe (Correa, Garcia, \& Garcia, 2018).

Por lo tanto, los Reportes Integrados se han transformado en un movimiento que promueve un nuevo formato de reporte corporativo, con énfasis en la revelación de aspectos claves, que den cuenta de la creación de valor por parte de las empresas (Navarrete \& Gallizo, 2018).

\section{Metodología}

La muestra del estudio está compuesta por las 19 principales empresas que cotizaban en la Bolsa de Valores de Colombia -BVC- según su capitalización en el mercado al 31 de diciembre de 2018. La elección de Colombia se debe al crecimiento económico que presenta el país latinoamericano en los últimos años, además de estar entre los cinco de países que más información sobre sostenibilidad emiten en sus reportes corporativos (Aristizábal, 2019). La BVC cuenta con 73 compañías emisores de títulos valores, de donde se tomaron 19 organizaciones que representan la mayor capitalización del mercado local, además sus actividades influyen en las prácticas de otras compañías y pueden tener mayor impacto en el mercado de capitales y en la economía doméstica.

Los datos se obtuvieron de los reportes publicados en las páginas web corporativas de cada una de las empresas seleccionadas, entre 2013 y 2018. Se consideró este periodo ya que la Global Reporting Initiative, publicó en 2014 la cuarta versión de su guía para la elaboración de los reportes de sostenibilidad, además en 2015 los líderes mundiales adoptaron los objetivos de desarrollo sostenible -ODS-. Por ende, este periodo de tiempo es relevante para observar como la adopción de diferentes estándares e iniciativas internacionales liderados por instituciones como la GRI, AccountAbility, IIRC, índice de sostenibilidad Dow Jones DJSI-, cambió la comunicación de la información no financiera a través de los reportes corporativos, que anteriormente utilizaban estándares como los Objetivos del Milenio, ISO 14001, ISO 26000.

Para el desarrollo de este estudio se utilizó la técnica de análisis de contenidos (Manuel et al., 2014; Rayrnond \& Luc Van, 2005), con el fin de analizar la evolución en las revelaciones de los reportes corporativos de las empresas objetos de estudio, es por ello que se llevó a cabo un análisis matricial en el cual se tomaron las categorías de análisis de manera categórica en donde tomaban el valor de uno (1) en caso de que existiera la revelación y cero (0) en otro caso, referenciado en trabajos previos como el de Sáez Martín et al. (2014). Las categorías de análisis consideradas en este estudio fueron seleccionadas con base en distintas investigaciones (Al Mubarak, Ben Hamed, \& Al Mubarak, 2019; Correa et al., 2018; Schönborn et al., 2019) y son: Características generales del reporte corporativo e información no financiera dentro de la cual se consideraron las revelaciones ambientales, revelaciones sociales, revelaciones económicas, revelaciones del gobierno corporativo y ODS.

El análisis se centra en cuatro tipos de reportes corporativos; Reporte Anual, Reporte Combinado, Reporte de Sostenibilidad y Reporte Integrado. Con los datos recopilados se llevaron a cabo 106 observaciones-año de los 114 totales. La lista de empresas seleccionadas se muestra en la tabla 1. 
TABLA 1

Composición de la muestra

\begin{tabular}{|c|c|c|c|}
\hline & Nombre & Sector & Ciudad \\
\hline 1 & Almacenes Éxito S. A. & Comercial & Envigado \\
\hline 2 & Avianca Holding S. A. & Industrial & Bogotá \\
\hline 3 & Banco Davivienda S. A & Financiero & Bogotá \\
\hline 4 & Banco de Bogotá S. A. & Financiero & Bogotá \\
\hline 5 & Bancolombia S. A. & Financiero & Medellin \\
\hline 6 & BVC & Financiero & Bogotá \\
\hline 7 & Canacol Energy Ltd. & Industrial & Bogotá \\
\hline 8 & Celsia & Industrial & Medellin \\
\hline 9 & Cemex Latan Holding & Industrial & Bogotá \\
\hline 10 & Constructora Conconcreto & Industrial & Medellin \\
\hline 11 & Corporación Financiera Colombiana S. A. & Financiero & Bogotá \\
\hline 12 & Ecopetrol S.A. & Industrial & Bogotá \\
\hline 13 & $\begin{array}{l}\text { Empresas de Telecomunicaciones de } \\
\text { Bogotá S. A }\end{array}$ & Telecomunicaciones & Bogotá \\
\hline 14 & Grupo Argos S. A & Inversiones & Medellin \\
\hline 15 & Grupo Aval & Inversiones & Bogotá \\
\hline 16 & Grupo de Energia de Bogotá & Inversiones & Bogotá \\
\hline 17 & Grupo de Inversiones SURA & Inversiones & Medellin \\
\hline 18 & Grupo Nutresa S. A & Inversiones & Medellin \\
\hline 19 & Isa S. A. & Industrial & Medellin \\
\hline
\end{tabular}

Fuente: elaboración propia.

En el análisis regional y sectorial se observa que las empresas se concentran en Medellín y Bogotá, con un $36,8 \%$ y $57,9 \%$ respectivamente, el sector industrial liderada la lista de las empresas con un $36,8 \%$, financiero 26,3\% e inversiones 21,1\% por otro lado, en el análisis combinado región-sector se encontró en Medellín mayor participación en el sector industrial e inversiones, en Bogotá predomina el sector industrial y financiero.

\section{Resultado y Discusiones}

\section{Evolución de las características generales del reporte corporativo}

En lo que respecta a las características generales del reporte corporativo se hace énfasis en los tipos de reporte y su nivel de adherencia al GRI. Las tablas 2 y 3 muestran de manera resumida la información acerca de la frecuencia respecto a los informes de las empresas estudiadas, en todos los periodos las organizaciones no publicaron algunos de los reportes antes mencionados y por ende se presentan algunos N/A.

TABLA 2

Frecuencia - Tipo de reporte por año

\begin{tabular}{|l|c|c|c|c|c|c|}
\hline Tipo de Reporte & 2013 & 2014 & 2015 & 2016 & 2017 & 2018 \\
\hline RA & 4 & 4 & 3 & 3 & 2 & 2 \\
\hline RC & 3 & 2 & 3 & 5 & 6 & 6 \\
\hline RS & 4 & 5 & 6 & 4 & 4 & 4 \\
\hline RI & 4 & 6 & 6 & 6 & 7 & 7 \\
\hline N/A & 4 & 2 & 1 & 1 & 0 & 0 \\
\hline Total & 19 & 19 & 19 & 19 & 19 & 19 \\
\hline
\end{tabular}

Fuente: elaboración propia. 
TABLA 3

Frecuencia Relativa - Tipo de reporte por año

\begin{tabular}{|l|c|c|c|c|c|c|}
\hline Tipo de Reporte & 2013 & 2014 & 2015 & 2016 & 2017 & 2018 \\
\hline RA & 0,21 & 0,21 & 0,16 & 0,16 & 0,11 & 0,11 \\
\hline RC & 0,16 & 0,11 & 0,16 & 0,26 & 0,32 & 0,32 \\
\hline RS & 0,21 & 0,26 & 0,32 & 0,21 & 0,21 & 0,21 \\
\hline RI & 0,21 & 0,32 & 0,32 & 0,32 & 0,37 & 0,37 \\
\hline N/A & 0,21 & 0,11 & 0,05 & 0,05 & - & - \\
\hline Total & 1,00 & 1,00 & 1,00 & 1,00 & 1,00 & 1,00 \\
\hline
\end{tabular}

Fuente: elaboración propia.

Tanto los reportes anuales como los de sostenibilidad presentan comportamientos decrecientes. Dado que las cifras financieras por si solas no muestran la generación de valor de las organizaciones, cada vez menos estos reportes son utilizados en el contexto colombiano. En contraste, el reporte combinado e integrado presentan las frecuencias relativas más altas, aunque el IR presenta una ventaja en comparación con el RC, centrado en su carácter holístico, que explica como las áreas mancomunadamente crean valor a la empresa en el corto, mediano y largo plazo. Esta tendencia hacia el reporte integrado es consistente con las tendencias a nivel global resaltadas en los estudios de Cortesi y Vena (2019), Havlová (2015), Pavlopoulos et al. (2019), Rupley et al. (2017).

Existen dos opciones para publicar información de acuerdo con los estándares GRI; Esencial o Exhaustivo (Global Sustainability Standard Board, 2016). La elección de uno u otro depende del alcance en la cantidad de información. Con el fin de analizar el comportamiento de adherencia al GRI en la muestra seleccionada, se establecieron tres categorías: Esencial, Exhaustivo y No aplica. Las tablas 4 y 5 resumen la información acerca de la cantidad y frecuencia en el nivel de adherencia al GRI. Las organizaciones que no revelaron información de acuerdo con los estándares emitidos por el GRI, que no definen su nivel de adherencia o utilizan otro estándar se agrupan en la categoría No aplica.

TABLA 4

Frecuencia - Nivel de adherencia GRI

\begin{tabular}{|l|c|c|c|c|c|c|}
\hline Nivel de Adherencia & $\mathbf{2 0 1 3}$ & $\mathbf{2 0 1 4}$ & $\mathbf{2 0 1 5}$ & $\mathbf{2 0 1 6}$ & $\mathbf{2 0 1 7}$ & $\mathbf{2 0 1 8}$ \\
\hline Exhaustiva & 2 & 4 & 3 & 2 & 2 & 2 \\
\hline Esencial & 7 & 9 & 11 & 12 & 13 & 13 \\
\hline No aplica & 10 & 6 & 5 & 5 & 4 & 4 \\
\hline Total & 19 & $\mathbf{1 9}$ & $\mathbf{1 9}$ & $\mathbf{1 9}$ & $\mathbf{1 9}$ & $\mathbf{1 9}$ \\
\hline
\end{tabular}

Fuente: elaboración propia.

TABLA 5

Frecuencia relativa - Nivel de adherencia GRI

\begin{tabular}{|l|c|c|c|c|c|c|}
\hline Nivel de Adherencia & $\mathbf{2 0 1 3}$ & $\mathbf{2 0 1 4}$ & $\mathbf{2 0 1 5}$ & $\mathbf{2 0 1 6}$ & $\mathbf{2 0 1 7}$ & $\mathbf{2 0 1 8}$ \\
\hline Exhaustiva & 0,11 & 0,21 & 0,16 & 0,11 & 0,11 & 0,11 \\
\hline Esencial & 0,37 & 0,47 & 0,58 & 0,63 & 0,68 & 0,68 \\
\hline No aplica & 0,53 & 0,32 & 0,26 & 0,26 & 0,21 & 0,21 \\
\hline \multicolumn{1}{|c|}{ Total } & $\mathbf{1 , 0 0}$ & $\mathbf{1 , 0 0}$ & $\mathbf{1 , 0 0}$ & $\mathbf{1 , 0 0}$ & $\mathbf{1 , 0 0}$ & $\mathbf{1 , 0 0}$ \\
\hline
\end{tabular}

Fuente: elaboración propia.

De acuerdo con la información presentada en las tablas 4 y 5 , el nivel de adherencia esencial suele ser el más utilizado por las organizaciones, con un total de 65 observaciones en los periodos examinados, con una frecuencia relativa promedio situada en $57 \%$, su tendencia es creciente debido al nivel de maduración presentado por las organizaciones en el concepto de sostenibilidad. El segundo lugar es ocupado por aquellas empresas que no definen su nivel de adherencia en el GRI o presentan un reporte de sostenibilidad bajo otro estándar, el total de observación es de 34, con una frecuencia relativa promedio del 30\%, su tendencia es decreciente por un menor uso de otros estándares diferentes al GRI y por las ventajas que puedan acarrear 
la publicación de información no financiera de las organizaciones, como aumento en la lealtad y mejora en la imagen corporativa (Gürlek et al., 2017). El nivel de adherencia Exhaustiva cuenta con 15 observaciones, las cuales se concentran en el sector industrial e inversiones, en organizaciones como Ecopetrol S.A y Grupo Nutresa S.A con seis y cinco observaciones respectivamente, que abarcan el 73,3\% de las observaciones totales. Esto evidencias una tendencia constante. Según lo establecido por Rodríguez y Ríos (2016), optar por publicar información con este nivel de adherencia requiere la evaluación de variables como el tamaño de la organización, el nivel de ventas, activos totales, tiempo y rentabilidad, aspectos que solo son satisfechas por unas cuantas organizaciones a nivel local.

\section{Información No Financiera}

\section{Evolución de las Revelaciones Ambientales}

La evolución de las revelaciones ambientales abarca aquellos indicadores publicados por las organizaciones con el objetivo de mostrar el progreso o retroceso de la información capturada de acuerdo con los tipos de informes. Los indicadores ambientales seleccionados concuerdan con la serie de estándares GRI clasificados en ocho categorías. La tabla 6 presenta la frecuencia de evolución por indicador y por año que han tenido las empresas estudiadas.

TABLA 6

Frecuencia-Evolución por Indicador Ambiental

\begin{tabular}{|l|c|c|c|c|c|c|c|c|}
\hline \multicolumn{1}{|c|}{ Aspecto } & $\begin{array}{c}\text { GRI } \\
\text { Estándar }\end{array}$ & $\mathbf{2 0 1 3}$ & $\mathbf{2 0 1 4}$ & $\mathbf{2 0 1 5}$ & $\mathbf{2 0 1 6}$ & $\mathbf{2 0 1 7}$ & $\mathbf{2 0 1 8}$ & Total \\
\hline Materiales & 301 & 18 & 14 & 21 & 17 & 15 & 16 & 101 \\
\hline Energia & 302 & 33 & 28 & 30 & 34 & 38 & 41 & 204 \\
\hline Agua & 303 & 18 & 16 & 19 & 22 & 24 & 26 & 125 \\
\hline Biodiversidad & 304 & 36 & 25 & 29 & 24 & 24 & 20 & 158 \\
\hline Emisiones & 305 & 43 & 63 & 60 & 67 & 74 & 75 & 382 \\
\hline Efluentes y Residuos & 306 & 25 & 15 & 22 & 27 & 32 & 29 & 150 \\
\hline Cumplimiento Ambiental & 307 & 16 & 11 & 13 & 11 & 15 & 12 & 78 \\
\hline $\begin{array}{l}\text { Evaluación ambiental de } \\
\text { Proveedores }\end{array}$ & 308 & 2 & 7 & 8 & 10 & 11 & 12 & 50 \\
\hline \multicolumn{1}{|c}{ Total } & & $\mathbf{1 9 1}$ & $\mathbf{1 7 9}$ & $\mathbf{2 0 2}$ & $\mathbf{2 1 2}$ & $\mathbf{2 3 3}$ & $\mathbf{2 3 1}$ & $\mathbf{1 2 4 8}$ \\
\hline
\end{tabular}

Fuente: elaboración propia.

Según se puede observar en la tabla 6, los indicadores con mayor frecuencia están relacionados con las categorías de Emisiones, Energía y Biodiversidad respectivamente. Resulta fundamental que las organizaciones manifiesten preocupación por la emisión de gases y su impacto directo al calentamiento global, especialmente las grandes compañías petroleras (Hirtenstein, 2017). Adicionalmente, los trabajos realizados por García et al. (2005) y Gürlek et al. (2017) muestran que la publicación de los impactos ambientales producido por las organizaciones independiente del sector, mejora la imagen corporativa y confianza por parte de los clientes.

Los indicadores relacionados con el consumo de energía presentan una tendencia positiva según la figura 1, por lo que se evidencia que las organizaciones buscan mejorar la eficiencia energética y reducir el importe de las inversiones, para minimizar los costos de producción, afectando también el precio final de los bienes y servicios (Ross, 1992). 


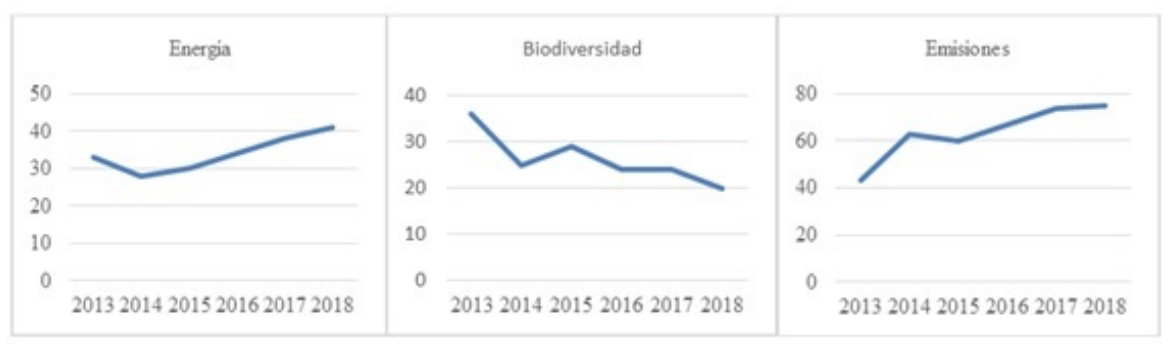

FIGURA 1

Indicadores ambientales de mayor variación en revelaciones

Fuente: elaboración propia.

La biodiversidad presenta un comportamiento decreciente en su revelación, lo cual es llamativo en razón de su alcance, donde abarca temas relacionados con la inversión y el gasto ambiental, la protección y restauración del habitad, número de árboles sembrados, entre otros. Fenómenos como el tráfico ilegal de fauna y flora, el cambio de clima, la deforestación y la realización o construcción de proyectos, amenazan la continuidad de muchas especies en el país, todo en consecuencia de un desarrollo que no ha sido bien planificado, además de la existencia de una única iniciativa efectiva hasta el momento para la conservación del habitad, "la declaración de área protegida" (González, 2017).

La figura 2 muestra que los temas ambientales dependen del sector económico de cada organización y que la actividad económica es una unidad de análisis determinante en la orientación de los resultados. El sector industrial e inversiones cuentan con el mayor número de indicadores ambientales publicados, 640 y 297 respectivamente. Esto se explica por la naturaleza propia del negocio donde participan las organizaciones. La industria financiera y comercial de la muestra seleccionada disminuye considerablemente la publicación de indicadores con 146 y 116 y el sector de las telecomunicaciones solo cuenta con 49 publicaciones.

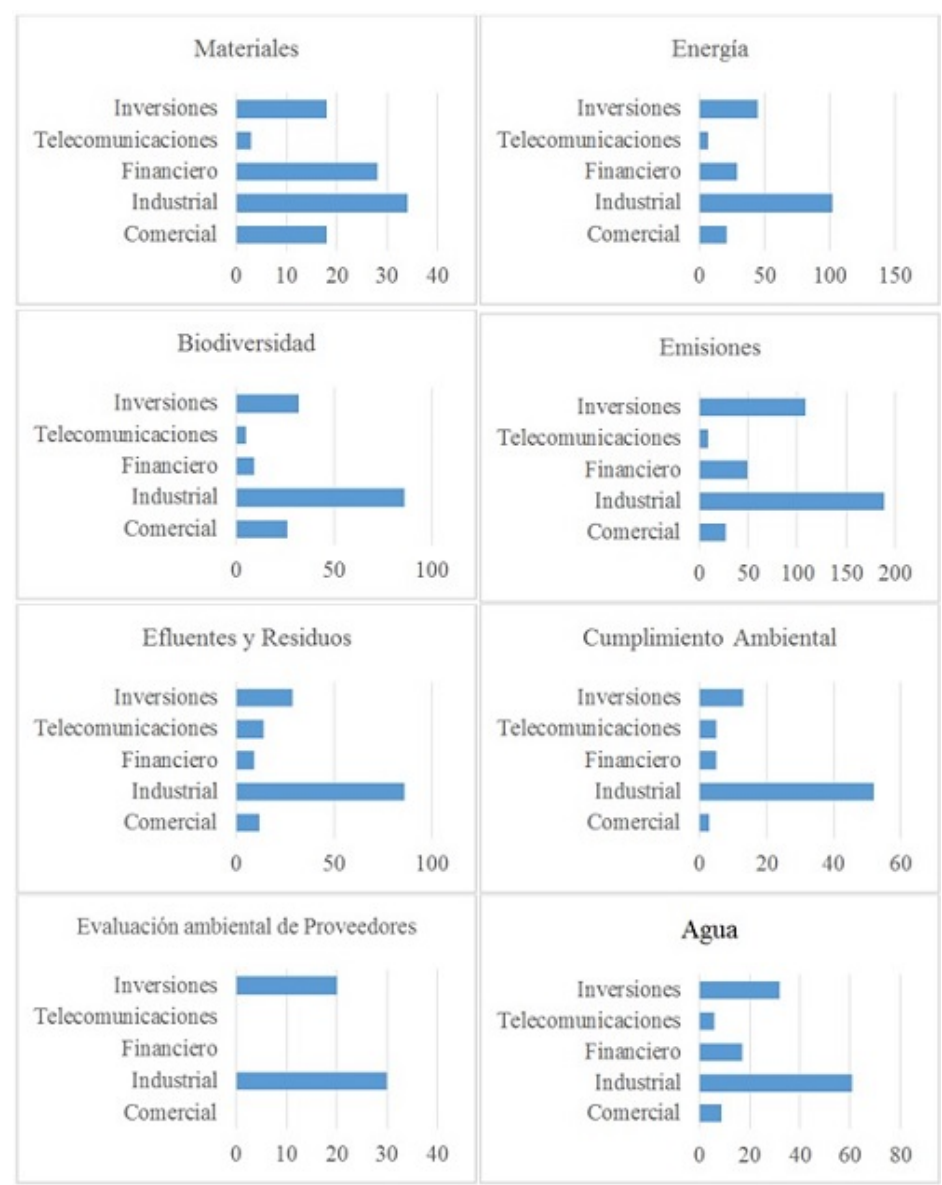

FIGURA 2

Frecuencia - Indicadores por Industria

Fuente: elaboración propia. 
Las revelaciones sobre el uso de los materiales están fuertemente implementadas en el sector industrial y el sector financiero. La revelación sobre el consumo de energía prevalece en el sector industrial, aunque es posible observar una fuerte participación del sector inversiones. Los indicadores relacionados con el uso del agua sobresalen en el sector industrial, al igual que los indicadores relacionados con el cuidado de la biodiversidad y efluentes y residuos, lo cual es consistente con el trabajo realizado por Babel et al. (2020). Por su parte, las emisiones, la evaluación ambiental de los proveedores y el cumplimiento ambiental son temas poco revelados en los sectores comercial, telecomunicaciones y financiero.

\section{Evolución de las Revelaciones Sociales}

Para el caso de los indicadores que miden el desempeño social de las organizaciones en los reportes corporativos se diseñó la tabla 7 , donde se presenta el porcentaje de revelación y no revelación de los aspectos sociales, la cual está codificada en la serie 400 y agrupados en 19 categorías.

\section{TABLA 7}

Frecuencia (\%) - Revelación y No Revelación Social

\begin{tabular}{|c|c|c|c|c|c|}
\hline Aspecto & $\begin{array}{c}\text { GRI } \\
\text { Estandar }\end{array}$ & $\begin{array}{c}\% \\
\text { Revelado }\end{array}$ & $\begin{array}{l}\% \text { No } \\
\text { Revelado }\end{array}$ & $\begin{array}{l}\text { Indicadores } \\
\text { revelados }\end{array}$ & Total \\
\hline Empleo & 401 & 0,52 & 0,48 & 170 & 330 \\
\hline $\begin{array}{l}\text { Relaciones entre los trabajadores y } \\
\text { la dirección }\end{array}$ & 402 & 0,31 & 0,69 & 34 & 110 \\
\hline Salud y seguridad en el trabajo & 403 & 0,37 & 0,63 & 162 & 440 \\
\hline Capacitación y educación & 404 & 0,56 & 0,44 & 184 & 330 \\
\hline Diversidad e igualdad de oportunidades & 405 & 0,44 & 0,56 & 96 & 220 \\
\hline No discriminación & 406 & 0,35 & 0,65 & 38 & 110 \\
\hline $\begin{array}{l}\text { Libertad de asociación y negociación } \\
\text { colectiva }\end{array}$ & 407 & 0,31 & 0,69 & 34 & 110 \\
\hline Trabajo infantil & 408 & 0,27 & 0,73 & 30 & 110 \\
\hline Trabajo forzoso & 409 & 0,26 & 0,74 & 29 & 110 \\
\hline Prácticas en materia de seguridad & 410 & 0,23 & 0,77 & 25 & 110 \\
\hline Derechos de la población indigena & 411 & 0,25 & 0,75 & 27 & 110 \\
\hline $\begin{array}{l}\text { Mecanismos de reclamación en materia } \\
\text { de derechos humanos }\end{array}$ & 412 & 0,22 & 0,78 & 122 & 550 \\
\hline Comunidades locales & 413 & 0,43 & 0,57 & 95 & 220 \\
\hline $\begin{array}{l}\text { Evaluación de las prácticas laborales } \\
\text { de los proveedores }\end{array}$ & 414 & 0,23 & 0,77 & 103 & 440 \\
\hline Politica pública & 415 & 0,22 & 0,78 & 190 & 880 \\
\hline Salud y seguridad de los clientes & 416 & 0,19 & 0,81 & 42 & 220 \\
\hline Marketing y etiquetado & 417 & 0,33 & 0,67 & 109 & 330 \\
\hline Privacidad de los clientes & 418 & 0,30 & 0,70 & 33 & 110 \\
\hline Cumplimiento regulatorio & 419 & 0,31 & 0,69 & 69 & 220 \\
\hline Total & & & & 1.592 & 5.060 \\
\hline
\end{tabular}

Fuente: elaboración propia.

Los indicadores con mayor porcentaje de revelación están relacionados con la educación y capacitación, empleo y diversidad e igualdad de oportunidades. Los indicadores que menos se revelaron fueron las de salud y seguridad de los clientes, mecanismos de reclamación en materia de derechos humanos y de política pública. El porcentaje de revelación de los demás indicadores oscilan entre el $23 \%$ y el $44 \%$, lo que da cuenta que los porcentajes de lo no revelado es en su gran mayoría superior al $50 \%$.

$\mathrm{Al}$ analizar los indicadores con mayor revelación se aprecia que tienen relación con el cumplimiento de algunos de los ODS. En concordancia con lo encontrado en este estudio, Geba \& Giusio (2017) observaron que tales indicadores tienen gran relación con los objetivos 4, 5 y 8 . Estos objetivos se enfocan en los grupos de interés, siendo los empleados uno de los más importantes, por lo que es relevante considerar el cumplimiento de tendencias actuales relacionadas con la estabilidad y oportunidades de aprendizaje (Archel, Husillos, \& Spence, 2011).

Los resultados sugieren que en la categoría social las entidades se acojan cada vez más a programas o políticas sociales para poder cumplir con los indicadores referenciados y así lograr aumentar el número de revelaciones y mejorar el desempeño social. La tabla 8 presenta la frecuencia de evolución por indicador y por año que han tenido los indicadores sociales. 
TABLA 8

Frecuencia - Evolución por indicadores sociales

\begin{tabular}{|c|c|c|c|c|c|c|c|c|}
\hline Aspecto & $\begin{array}{c}\text { GRI } \\
\text { Estándar }\end{array}$ & 2013 & 2014 & 2015 & 2016 & 2017 & 2018 & Total \\
\hline Empleo & 401 & 21 & 29 & 33 & 28 & 29 & 30 & 170 \\
\hline $\begin{array}{l}\text { Relaciones entre los trabajadores y } \\
\text { la dirección }\end{array}$ & 402 & 6 & 7 & 6 & 5 & 5 & 5 & 34 \\
\hline Salud y seguridad en el trabajo & 403 & 19 & 24 & 36 & 25 & 29 & 29 & 162 \\
\hline Capacitación y educación & 404 & 19 & 28 & 36 & 32 & 32 & 37 & 184 \\
\hline Diversidad e igualdad de oportunidades & 405 & 13 & 14 & 18 & 17 & 15 & 19 & 96 \\
\hline No discriminación & 406 & 5 & 8 & 8 & 6 & 5 & 6 & 38 \\
\hline $\begin{array}{l}\text { Libertad de asociación y negociación } \\
\text { colectiva }\end{array}$ & 407 & 6 & 7 & 5 & 5 & 7 & 4 & 34 \\
\hline Trabajo infantil & 408 & 5 & 5 & 6 & 5 & 5 & 4 & 30 \\
\hline Trabajo forzoso & 409 & 5 & 5 & 6 & 5 & 4 & 4 & 29 \\
\hline Prácticas en materia de seguridad & 410 & 5 & 4 & 7 & 3 & 3 & 3 & 25 \\
\hline Derechos de la población indigena & 411 & 4 & 6 & 6 & 4 & 3 & 4 & 27 \\
\hline $\begin{array}{l}\text { Mecanismos de reclamación en materia } \\
\text { de derechos humanos }\end{array}$ & 412 & 21 & 27 & 33 & 20 & 11 & 10 & 122 \\
\hline Comunidades locales & 413 & 15 & 20 & 19 & 14 & 14 & 13 & 95 \\
\hline $\begin{array}{l}\text { Evaluación de las prácticas laborales } \\
\text { de los proveedores }\end{array}$ & 414 & 25 & 22 & 27 & 19 & 5 & 5 & 103 \\
\hline Politica pública & 415 & 40 & 51 & 54 & 26 & 10 & 9 & 190 \\
\hline Salud y seguridad de los clientes & 416 & 9 & 8 & 9 & 7 & 5 & 4 & 42 \\
\hline Marketing y etiquetado & 417 & 26 & 27 & 27 & 16 & 5 & 8 & 109 \\
\hline Privacidad de los clientes & 418 & 5 & 6 & 7 & 5 & 4 & 6 & 33 \\
\hline Cumplimiento regulatorio & 419 & 9 & 12 & 16 & 12 & 9 & 11 & 69 \\
\hline Total & & 258 & 310 & 359 & 254 & 200 & 211 & 1.592 \\
\hline
\end{tabular}

Fuente: elaboración propia.

El comportamiento a través de los años de los indicadores que fueron pioneros en el porcentaje de revelación mantiene un crecimiento en sus revelaciones, dado que las empresas trabajan en proporcionar información que ayude a medir su desempeño social con respecto a dichas dimensiones. Por el contrario, la Política pública, Evaluación de las prácticas laborales de los proveedores y Marketing y etiquetado, presentan un comportamiento descendente en el número de revelaciones.

Con respecto al indicador de política pública, las empresas tardan en brindar información acerca de sus vínculos con los procesos políticos del país, dado que las contribuciones directas o indirectas pueden presentar riesgos de corrupción. En este sentido, es posible que dichos vínculos políticos sean vistos por algunas empresas como un obstáculo al desarrollo de sus actividades, debido al riesgo de corrupción al que se someterían (Fernández \& Ramón, 2013).

En cuanto a los indicadores de evaluación de las prácticas laborales de los proveedores y Marketing y etiquetado, Fernández y Ramón (2013) observaron que las empresas deben de tener un canal de comunicación de marketing justo y responsable, donde los clientes y usuarios finales tengan acceso a la información adecuada sobre lo positivo y lo no tan positivo de los impactos ambientales y sociales de los productos y servicios con el fin de ayudar a los clientes a tomar decisiones informadas. Proporcionar dicha información puede estar vinculado con el cumplimiento de ciertos tipos de regulaciones, leyes y directrices, como las de la OCDE (Mesa, 2017).

Referente a la evaluación de las prácticas de los proveedores, podrían mejorar las prácticas de adquisición, las expectativas de desempeño y analizar los posibles impactos sociales negativos en la cadena de suministro (Fernández \& Ramon, 2013; Mesa, 2017). La poca revelación de información que tenga que ver con los mencionados indicadores puede indicar una gestión interna inadecuada o un deterioro en la efectividad de los controles internos.

\section{Evolución de las Revelaciones Económicas}

La evolución de las revelaciones económicas abarca aquellos indicadores publicados por las organizaciones con el objetivo de mostrar impactos significativos en cuanto a temas económicos, su progreso o retroceso de la información capturada por los tipos de informes. Los indicadores económicos seleccionados son la serie 
200 de los estándares GRI clasificados en seis categorías. La tabla 9 presenta la frecuencia de evolución por indicador y por año.

TABLA 9

Frecuencia - Evolución por indicador económico

\begin{tabular}{|l|c|c|c|c|c|c|c|c|}
\hline \multicolumn{1}{|c|}{ Aspecto } & $\begin{array}{c}\text { GRI } \\
\text { Estándar }\end{array}$ & $\mathbf{2 0 1 3}$ & $\mathbf{2 0 1 4}$ & $\mathbf{2 0 1 5}$ & $\mathbf{2 0 1 6}$ & $\mathbf{2 0 1 7}$ & $\mathbf{2 0 1 8}$ & Total \\
\hline Desempeño económico & 201 & $\mathbf{2 4}$ & $\mathbf{2 7}$ & 27 & $\mathbf{2 5}$ & $\mathbf{2 5}$ & $\mathbf{2 2}$ & 150 \\
\hline Presencia en el mercado & 202 & 11 & 11 & 9 & 10 & 9 & 10 & 60 \\
\hline $\begin{array}{l}\text { Impactos Económicos } \\
\text { Indirectos }\end{array}$ & 203 & 14 & 12 & 10 & 10 & 15 & 14 & 75 \\
\hline Prácticas de adquisición & 204 & 8 & 8 & 10 & 9 & 10 & 10 & 55 \\
\hline Anticomupción & 205 & 0 & 0 & 0 & 11 & 27 & 29 & 67 \\
\hline Competencia desleal & 206 & 0 & 0 & 0 & 3 & 5 & 7 & 15 \\
\hline \multicolumn{1}{|c|}{ Total } & & $\mathbf{5 7}$ & $\mathbf{5 8}$ & $\mathbf{5 6}$ & $\mathbf{6 8}$ & $\mathbf{9 1}$ & $\mathbf{9 2}$ & $\mathbf{4 2 2}$ \\
\hline
\end{tabular}

Fuente: elaboración propia

Los indicadores de desempeño económico, impactos económicos directos y anticorrupción tienen la mayor cantidad de revelaciones. El desempeño económico exhibe una tendencia constante lo cual se explica por el tipo de información mostrada en las organizaciones, donde se reflejan los ingresos de las operaciones, ventas de activos, gastos por funcionamiento, sueldos, pago a accionistas. Información de índole financiero relacionado normalmente en los estados financieros.

Los impactos económicos indirectos se centran en la inversión en infraestructura y servicios e impactos económicos indirectos significativos. Su comportamiento es constante, como se aprecia en la figura 3, e involucra líneas de inversión relacionadas con la educación, salud, infraestructura comunitaria, promoción social, etc. (Pavlopoulos et al., 2019). La inversión social, al igual que el desempeño económico, se muestra constante pero no revela la cantidad de dinero utilizado por las organizaciones en sus proyectos. Es importante resaltar en el caso de Colombia, el posconflicto y la migración venezolana constituyen fenómenos que requieren importante inversión social, apoyada por empresas como ISA, Grupo Nutresa, Grupo Argos, Ecopetrol (Revista Dinero, 2019).

Las revelaciones relacionadas con temas de anticorrupción, tiene uno de los mayores crecimientos en la cantidad de publicaciones por año. Según la Association of Certified Fraud Examiners (ACFE, 2018), en 2018 Colombia era el tercer país con mayor corrupción interna en sus empresas. Se espera que las organizaciones pretendan mostrar las herramientas utilizadas para contrarrestar este delito, y evitar la repetición de casos como; Odebrecht, Interbolsa, carrusel de la contratación, SaludCoop, etc.

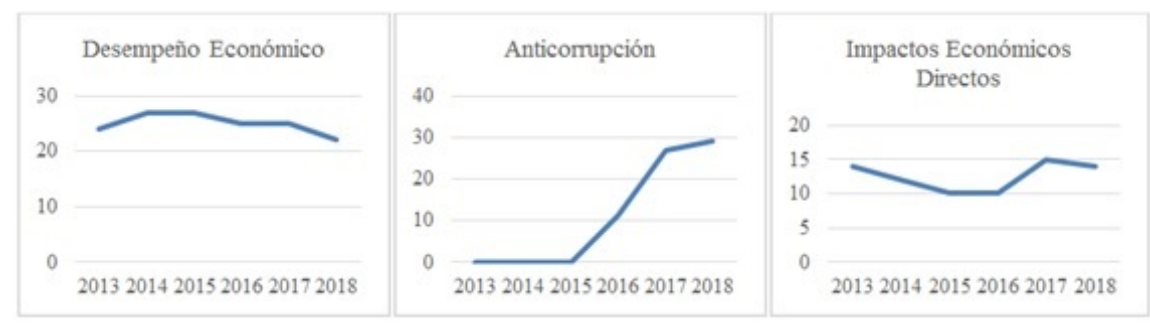

FIGURA 3

Indicadores Económicos con mayor revelación entre 2013 y 2018

Fuente: elaboración propia.

\section{Evolución de Revelaciones del Gobierno Corporativo}

Esta categoría está identificada dentro del itinerario de la guía del GRI en la serie 102, desde el numeral 18 hasta el 39 en el cual se abarca distintos indicadores como los identificados en la tabla 10 que corresponden a revelaciones generales sobre la empresa que informa. 
TABLA 10

Frecuencia - Evolución por indicador del gobierno corporativo

\begin{tabular}{|c|c|c|c|c|c|c|c|}
\hline $\mathrm{N}^{\circ}$ & Indicador gobierno corporativo & 2013 & 2014 & 2015 & 2016 & 2017 & 2018 \\
\hline 18 & Estructura de gobernanza & 9 & 12 & 14 & 13 & 14 & 15 \\
\hline 19 & Delegación de autoridad & 0 & 7 & 7 & 8 & 7 & 10 \\
\hline 20 & $\begin{array}{l}\text { Responsabilidad a nivel ejecutivo de temas } \\
\text { económicos, ambientales y sociales }\end{array}$ & 7 & 10 & 9 & 8 & 9 & 12 \\
\hline 21 & $\begin{array}{l}\text { Consulta a grupos de interés sobre temas } \\
\text { económicos, ambientales y sociales }\end{array}$ & 4 & 8 & 7 & 7 & 6 & 8 \\
\hline 22 & $\begin{array}{l}\text { Composición del máximo órgano de gobierno } \\
\text { y sus comités }\end{array}$ & 7 & 11 & 10 & 10 & 10 & 12 \\
\hline 23 & Presidente del máximo órgano de gobierno & 7 & 10 & 11 & 11 & 11 & 14 \\
\hline 24 & $\begin{array}{l}\text { Nominación y selección del máximo órgano } \\
\text { de gobierno }\end{array}$ & 6 & 10 & 11 & 10 & 10 & 11 \\
\hline 25 & Conflictos de intereses & 6 & 11 & 11 & 12 & 11 & 12 \\
\hline 26 & $\begin{array}{l}\text { Función del máximo órgano de gobiemo en la } \\
\text { selección de objetivos, valores y estrategia }\end{array}$ & 5 & 9 & 10 & 9 & 8 & 11 \\
\hline 27 & $\begin{array}{l}\text { Conocimientos colectivos del máximo órgano } \\
\text { de gobierno }\end{array}$ & 5 & 9 & 8 & 8 & 7 & 8 \\
\hline 28 & $\begin{array}{l}\text { Evaluación del desempeño del máximo } \\
\text { órgano de gobierno }\end{array}$ & 5 & 9 & 11 & 9 & 8 & 8 \\
\hline 29 & $\begin{array}{l}\text { Identificación y gestión de impactos } \\
\text { económicos, ambientales y sociales }\end{array}$ & 5 & 9 & 9 & 9 & 8 & 10 \\
\hline 30 & Eficacia de los procesos de gestión del riesgo & 5 & 9 & 10 & 9 & 9 & 11 \\
\hline 31 & $\begin{array}{l}\text { Evaluación de temas económicos, } \\
\text { ambientales y sociales }\end{array}$ & 6 & 11 & 9 & 8 & 8 & 8 \\
\hline 32 & $\begin{array}{l}\text { Función del máximo órgano de gobiemo en la } \\
\text { elaboración de informes de sostenibilidad }\end{array}$ & 7 & 10 & 10 & 9 & 10 & 12 \\
\hline 33 & Comunicación de preocupaciones críticas & 4 & 8 & 8 & 8 & 7 & 9 \\
\hline 34 & $\begin{array}{l}\text { Naturaleza y número total de preocupaciones } \\
\text { eriticas }\end{array}$ & 0 & 7 & 7 & 7 & 7 & 7 \\
\hline 35 & Politicas de remuneración & 6 & 9 & 9 & 9 & 9 & 10 \\
\hline 36 & Proceso para determinar la remuneración & 6 & 9 & 9 & 7 & 8 & 10 \\
\hline 37 & $\begin{array}{l}\text { Involucramiento de los grupos de interés en la } \\
\text { remuneración }\end{array}$ & 0 & 7 & 7 & 7 & 7 & 9 \\
\hline 38 & Ratio de compensación total anual & 0 & 3 & 2 & 2 & 2 & 3 \\
\hline 39 & $\begin{array}{l}\text { Ratio del incremento porcentual de la } \\
\text { compensación total anual }\end{array}$ & 0 & 3 & 3 & 3 & 2 & 3 \\
\hline
\end{tabular}

Fuente: elaboración propia.

Para el análisis de los resultados se clasificaron los indicadores de la tabla 10 en una única categoría de análisis, identificada como Gobierno Corporativo con código 102, cuyo objetivo según el GRI es proporcionar información contextual que sea relevante de la organización que prepara informes de sostenibilidad bajo el enfoque de la guía del GRI, en este caso sobre la gobernanza de la entidad que informa.

Las revelaciones en esta sección dan una visión general de la estructura de gobierno corporativo y su composición, el papel del máximo órgano de gobierno en cuanto a la definición del propósito, los valores y la estrategia de la organización, en la gestión de riesgos, en la presentación de informes de sostenibilidad, en la evaluación del desempeño económico, ambiental y social; en las competencias y la evaluación del desempeño y en la remuneración e incentivos del mismo. En lo que respecta a los indicadores relacionados con la remuneración, fueron los que menos revelaciones presentaron, debido a que en el transcurso de la búsqueda de la información se identificó que las entidades no reportaban dichos indicadores por motivos de seguridad y confidencialidad del personal clave de las entidades, lo cual es coherente con lo expuesto por Verrecchia (1983).

El análisis general de los demás indicadores revela un comportamiento estable en el tiempo, resaltando un ligero aumento en el año 2018, lo cual denota el interés de las empresas por publicar información relacionada con la gobernanza de la entidad.

\section{Objetivos de Desarrollo Sostenible (ODS)}

Otro de los aspectos analizados en la investigación es el compromiso que tienen las entidades con los ODS. En cada reporte se identificaron los ODS que fueron priorizados por las empresas durante los años 2015-2018. La tabla 11 presenta la frecuencia de evolución por ODS y por año que han tenido las empresas estudiadas. 
TABLA 11

Objetivos de Desarrollo Sostenible priorizados por año

\begin{tabular}{|l|c|c|c|c|c|}
\hline \multicolumn{1}{|c|}{ Objetivos de Desarrollo Sostenible } & $\mathbf{2 0 1 5}$ & $\mathbf{2 0 1 6}$ & $\mathbf{2 0 1 7}$ & $\mathbf{2 0 1 8}$ & $\begin{array}{c}\text { Frecuencia } \\
\text { Relativa }\end{array}$ \\
\hline 1. Fin de la Pobreza & 4 & 6 & 7 & 5 & 0,06 \\
\hline 2. Hambre Cero & 3 & 4 & 5 & 4 & 0,04 \\
\hline 3. Salud y Bienestar & 3 & 6 & 7 & 5 & 0,05 \\
\hline 4. Educación de Calidad & 4 & 6 & 11 & 11 & 0,08 \\
\hline 5. Igualdad de Genero & 3 & 5 & 6 & 5 & 0,05 \\
\hline 6. Agua Limpia y Saneamiento & 3 & 5 & 6 & 6 & 0,05 \\
\hline 7. Energia Asequible y no Contaminante & 3 & 6 & 7 & 9 & 0,06 \\
\hline 8. Trabajo decente y Crecimiento Económico & 3 & 8 & 12 & 10 & 0,08 \\
\hline 9. Industria Innovación e Infraestructura & 3 & 6 & 8 & 11 & 0,07 \\
\hline 10. Reducción de las Desigualdades & 3 & 5 & 6 & 6 & 0,05 \\
\hline 11. Ciudades y Comunidades Sostenibles & 3 & 7 & 8 & 8 & 0,07 \\
\hline 12. Producción y Consumo Responsable & 3 & 6 & 9 & 9 & 0,07 \\
\hline 13. Acción por el Clima & 4 & 7 & 9 & 12 & 0,08 \\
\hline 14. Vida Submarina & 3 & 2 & 2 & 3 & 0,03 \\
\hline 15. Vida de Ecosistemas Terrestres & 4 & 6 & 6 & 5 & 0,05 \\
\hline 16. Paz, Justicia e Instituciones Sólidas & 4 & 7 & 9 & 8 & 0,07 \\
\hline 17. Alianzas para lograr los objetivos & 2 & 5 & 6 & 7 & 0,05 \\
\hline \multicolumn{1}{|c|}{ Total } & 55 & 97 & 124 & 124 & 1,00 \\
\hline
\end{tabular}

Fuente: elaboración propia.

Los ODS con mayor priorización son los objetivos 4, 8 y 13. Por el contrario, el menos priorizado fue el 14 con una frecuencia relativa de 0,03 . En un principio explicado por la naturaleza de las empresas objeto de estudio y ubicación, puesto que se encuentran en las principales ciudades de Colombia y su operación no tiene impacto directo en los océanos.

Se puede establecer que la mayoría de empresas ( $73 \%$ aproximadamente), están comprometidas con al menos un ODS que abarca cada aspecto de la sociedad. Sin embargo, se considera que aún falta compromiso por parte de las entidades con los ODS, dado que hay algunos objetivos que se consideran de primera necesidad y que tienen baja adopción por parte de las empresas, como el fin de la pobreza, del hambre y desigualdades, siendo estos representados en los objetivos 1, 2 y 10 . Hay que tener en cuenta que estos objetivos fueron diseñados con la premisa que son acuerdos jurídicamente no vinculantes, es decir, acuerdos promovidos sin responsabilidades precisas para las empresas, lo que facilita el incumplimiento y el escepticismo que generan en varios sectores (Sanahuja \& Tezanos, 2017).

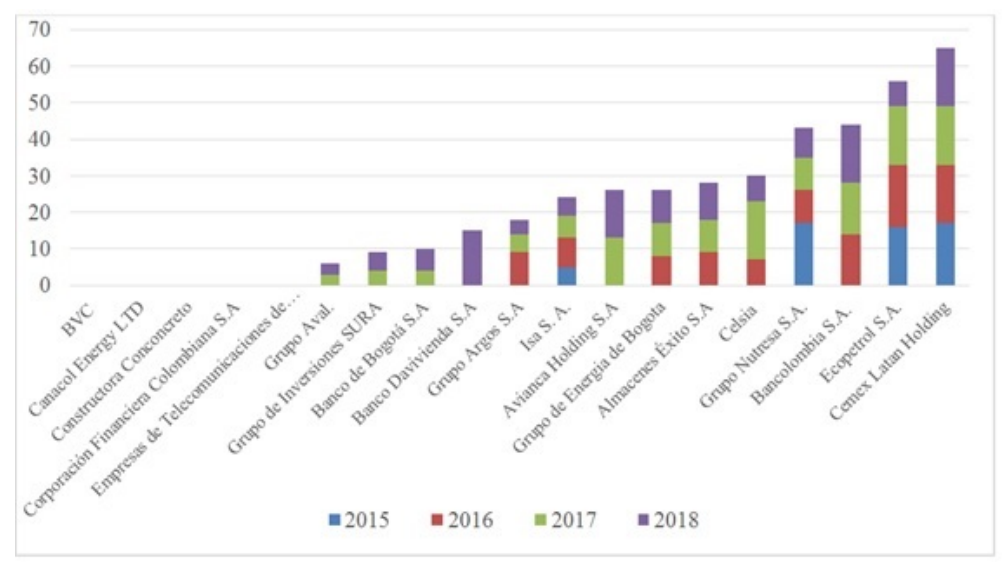

FIGURA 4

ODS priorizados por empresa

Fuente: elaboración propia.

Las empresas que más se destacaron por su compromiso con los ODS, son Cemex y Ecopetrol con un total de 65 y 56 revelaciones respectivamente durante los años estudiados. Además, Isa, Grupo Nutresa y Ecopetrol fueron las entidades pioneras en la adopción de los objetivos, ya que fueron las únicas que desde el 2015 iniciaron con el proceso de adopción de los ODS. Sin embargo, hay cinco empresas pertenecientes a 
varios sectores que no manifestaron en los reportes corporativos el compromiso con los ODS en ninguno de los años, empresas como BVC, Canacol, la Corporación Financiera, Constructora Conconcreto y Empresas de Telecomunicaciones de Bogotá.

\section{Conclusiones}

Este estudio se centró en tres componentes: las revelaciones generales de los reportes corporativos, información no financiera evaluada según estándares internacionales como lo es el GRI y los ODS. En cuanto a los reportes corporativos; los reportes anuales constituyen una práctica que ha disminuido durante el tiempo por el aumento en la publicación de reporte combinado e integrado en las empresas, seguido del informe de sostenibilidad. Adicionalmente el nivel de adherencia al GRI en su opción esencial ha sido el más usado durante los últimos años.

En cuanto a la información no financiera de los reportes, se presentan avances importantes en la cantidad de información revelada por las empresas en ciertas categorías de análisis estudiadas (ambiental. social, económico y gobierno corporativo). En la categoría ambiental se destacó la importancia que tienen para las empresas los indicadores de emisión, agua y biodiversidad; en la categoría social, los indicadores pioneras son las de capacitación y educación, empleo y por último la diversidad e igualdad de oportunidades, en lo económico los indicadores de mayor revelación fueron los de desempeño económico, anticorrupción e impactos económicos directos, y finalmente se encontró un nivel de revelaciones relativamente estables en el tiempo en los indicadores del gobierno corporativo. Así pues, se presentan en las categorías mencionadas una evolución en la cantidad de información revelada, que respaldan las expectativas de las empresas, en especial de los Stakeholders por generar valor, resaltando los consumidores, competidores y las organizaciones determinantes en el comportamiento responsable de estas (Benlemlih, 2019; Park \& Cave, 2018). Pero también se presentaron indicadores con retroceso en el nivel de revelación que se pudieron ver afectadas de acuerdo con el tipo de mensaje (Robinson \& Eilert, 2018).

En cuanto al promedio de revelación y no revelación, los indicadores relacionados con el gobierno corporativo ocupan el primer lugar con un promedio de 44\%, lo cual presenta una relación fuerte con los resultados de Sáez Martín et al. (2014), los temas sociales se ubican en el segundo lugar con un promedio de $32 \%$, en tercer lugar, se encuentra los indicadores económicos con un $20 \%$, y por último los indicadores ambientales con un 9\%, lo que permite inferir el tipo de información más elocuente a revelar en el contenido de los reportes corporativos de las compañías que conformaban la muestra.

El análisis indica que existen diferencias y particularidades importantes entre los informes, en contenido, extensión, redacción, etc. publicando indicadores comunes con alta importancia e interés social. Del mismo modo, las motivaciones corporativas atribuidas al trabajo decente y educación de calidad, inmersas en los objetivos de desarrollo sostenible, impulsan la imagen corporativa, como lo han encontrado otros estudios (Al Mubarak et al., 2019; Sahin \& Bayramoğlu, 2015).

La relevancia de este artículo se centra en sus implicaciones puesto que mejora la comprensión de la información para aquellas empresas que requieren revelar información no financiera, además de formular nuevos interrogantes con respecto a los tipos de reportes por industria. Desde el punto de vista académico, el artículo aborda la problemática de la discrecionalidad de información, en la práctica la discrecionalidad de información es fundamental para la toma de decisiones empresariales. Desde el punto de vista legal, el alcance se ve limitado por la voluntariedad de adoptar los tipos de reportes con información no financiera (Rodríguez \& Ríos, 2016).

Las principales limitantes que se afrontaron para el desarrollo de la investigación surgieron en la selección de la muestra, ya que en el índice de la BVC no se tiene información de empresas de diversos sectores, igualmente las empresas no publicaron en todos los periodos estudiados los reportes corporativos que eran 
esenciales, incluso se tuvo en cuenta la autonomía de cada empresa en el diseño de su formato para la presentación de la información.

Las organizaciones se representan receptivas a las necesidades y preocupaciones de la sociedad, de manera que los reportes corporativos juegan un papel estratégico y de interés que motiva su estudio por parte de la academia, estado y empresas. En este sentido, el artículo abre la posibilidad de desarrollo nuevas investigaciones académicas enfocadas a la publicación de reportes corporativos por tipo de industria, relación que ha sido poco explorada en el ámbito local, por ende, dicho estudio es precursor en el campo de investigación.

\section{Referencias}

Abeysekera, I. (2013). A template for integrated reporting. Journal of Intellectual Capital,14(2), 227-245. https://do i.org/10.1108/14691931311323869

ACFE (2018). Report to the nations; 2018 Global Study on Occupational Fraud and Abuse; Latin America and the Caribbean Edition. Recuperado de: https://www.acfe.com/uploadedFiles/ACFE_Website/Content/rttn/201 8/RTTN-Latin-America-and-the-Caribbean.pdf

Acosta, P., \& Pérezts, M. (2019). Unearthing Sedimentation Dynamics in Political CSR: The Case of Colombia. Journal of Business Ethics, 155(2), 425-444. https://doi.org/10.1007/s10551-017-3502-x

Al Mubarak, Z., Ben Hamed, A., \& Al Mubarak, M. (2019). Impact of corporate social responsibility on bank's corporate image. Social Responsibility Journal, 15(5), 710-722. https://doi.org/10.1108/SRJ-01-2018-0015

Archel, P., Husillos, J., \& Spence, C. (2011). The institutionalisation of unaccountability: Loading the dice of Corporate Social Responsibility discourse. Accounting, Organizations and Society, 36(6), 327-346. https://doi. org/10.1016/j.aos.2011.06.003

Aristizábal, M. (30 de Julio de 2019). Un reporte de sostenibilidad debe ser conciso y comparable. La República. Recuperado de: https://www.larepublica.co/especiales/especial-rse-julio-2019/un-reporte-de-sostenibilidad-d ebe-ser-conciso-y-comparable-tim-mohin-2890722

Ashforth, B. E. (2014). The Double-Edge of Organizational Legitimation.Organization science, 1, 177-194. https:// doi.org/10.1287/orsc.1.2.177

Austin, L., \& Gaither, B. M. (2017). Perceived motivations for corporate social responsibility initiatives in socially stigmatized industries. Public Relations Review, 43(4), 840-849. https://doi.org/10.1016/j.pubrev.2017.06.011

Babel, M., Oo, E., Shinde, V., Kamalamma, A., \& Haarstrick, A. (2020). Comparative study of water and energy use in selected automobile manufacturing industries. Journal of Cleaner Production, 246, 118970. https://doi.org/ 10.1016/j.jclepro.2019.118970

Ballou, B., Chen, P. C., Grenier, J. H., \& Heitger, D. L. (2018). Corporate social responsibility assurance and reporting quality: Evidence from restatements. Journal of Accounting and Public Policy, 37(2), 167-188. https://doi.org/ 10.1016/j.jaccpubpol.2018.02.001

Benlemlih, M. (2019). Corporate social responsibility and dividend policy. Research in International Business and Finance, 47, 114-138. https://doi.org/10.1016/j.ribaf.2018.07.005

Correa, J, Garcia, M., \& Garcia E. (2018). CSR Communication strategies of Colombian business groups: An analysis of corporate reports. Sustainability, 10, 1602. https://doi:10.3390/su10051602

Cortesi, A., \& Vena, L. (2019). Disclosure quality under Integrated Reporting: A value relevance approach. Journal of Cleaner Production, 220, 745-755. https://doi.org/10.1016/j.jclepro.2019.02.155

Cuevas, J., Maldonado, S., \& Escobar, N. (2013). Aproximación a los factores que influyen en la divulgación de información sobre RSC en empresas de América Latina. Cuadernos de Contabilidad, 14(34), 91-131.

Dávila, A., Rodríguez, C., \& Elvira, M. (2018). Engaging Stakeholders in Emerging Economies: The Case of Multilatinas. Journal of Business Ethics, 152(4), 949-64. https://doi.org/10.1007/s10551-018-3820-7 
DiMaggio, P. J., \& Powell, W. W. (1983). The Iron Cage Revisited: Institutional Isomorphism and Collective Rationality in organizational fields. American Sociological Review, 48(2), 147-160. https://doi.org/10.2307/20 95101

Dragu, I. M., \& Tiron-Tudor, A. (2013a). The integrated reporting initiative from an institutional perspective: emergent factors. Procedia-Social and Behavioral Sciences, 92, 275-279. https://doi.org/10.1016/j.sbspro.2013 .08 .672

Dragu, I. M., \& Tiron-Tudor, A. (2013b). New corporate reporting trends analysis on the evolution of integrated reporting. Journal The Annals of the University of Oradea. Economic Sciences, 22, 1221. https://ideas.repec.org/ a/ora/journl/v1y2013i1p1221-1228.html

Duran, D. C., Artene, A., Gogan, L. M., \& Duran, V. (2015). The Objectives of Sustainable Development - Ways to Achieve Welfare. Procedia Economics and Finance, 26(15), 812-817.https://doi.org/10.1016/s2212-5671(15)0 0852-7

Eccles, R. G. \& Krzus, M. P. (2010). One report. Integrated reporting for a sustainability strategy. Hoboken, New Jersey. John Wiley and Sons, Inc. https://books.google.es/books?hl=es\&lr=\&id=mEBe9MBOWn4C\&oi=fnd\&pg= PT9\&dq=One+report. + Integrated + reporting + for $+a+$ sustainability + strategy\&ots $=\mathrm{KxmoKmHs}$ i i\&sig=tO vCQpxbqEtjkfbgWgKJbIN_wCw\#v=onepage\&q=One\%20report.\%20Integrated\%20reporting\%20for\%20a \%20sustainability\%20strategy\& $\mathrm{f}=$ false

Eccles, R. G. \& Saltzman, D. (2010). Achieving Sustainability through Integrated Reporting. Stanford Social Innovation Review. 9(3), 56-61. https://www.hbs.edu/faculty/Pages/item.aspx?num=40617

Fernández, E., \& Ramón, R. (2013). Revelación de la información financiera y no financiera: Informes Integrados. Revista Contable, 10,78-86. https://rua.ua.es/dspace/bitstream/10045/42002/1/2013_Fernandez_Ramon_R evistaContable_2.pdf

Fernández, J. L., \& Bajo, A. (2012). La Teoría del Stakeholder o de los Grupos de Interés, pieza clave de la RSE, del éxito empresarial y de la sostenibilidad. Revista Internacional de Investigación en Comunicación, 6(6), 130-143. https://doi:10.7263/ADR.RSC.006.07

Freeman, E., \& McVea, J. (1984). A stakeholder approach to strategic management. working paper No. 01-02. Darden Business School, 01, 1-32. https://doi.org/10.2139/ssrn.263511

Freeman, R. E. (1984). Strategic management: A stakeholder approach. In Freeman Edward (Vol. 1). https://doi.or $\mathrm{g} / 10.2139 / \mathrm{ssrn} .263511$

Freundlieb, M., \& Teuteberg, F. (2013). Informes de responsabilidad social corporativa: un análisis transnacional de los informes de responsabilidad social corporativa en línea de empresas que cotizan en bolsa: los contenidos y su evolución. International Journal of Innovation and Sustainable Development, 7(1), 1-26. https://doi.org/10.15 04/IJISD.2013.052117

García, M., Rodríguez, I., \& Herrero, A. (2005). Influence of Corporate Social Responsibility on Loyalty and Valuation of Services. Journal of Business Ethics, 61, 369-385. https://doi.org/10.1007/s10551-005-5841-2

Geba, N. B., \& Giusio, M. (2017). Contabilidad social y ambiental: los estándares GRI 2016 y los objetivos del desarrollo sustentable. Simposio llevado a cabo en el XXXVIII Jornadas Universitarias de Contabilidad (Universidad Nacional del Sur, Bahía Blanca, 15 al 17 de noviembre de 2017) http://sedici.unlp.edu.ar/handl e/10915/72810

Global Sustanability Standars Board. (2016). Gri 102: contenidos generales 2016. Recuperado de: https://www.globa lreporting.org/standards/media/1440/spanish-gri-102-general-disclosures-2016.pdf

González, E. (2007). La teoría de los Stakeholders: Un puente para el desarrollo práctico de la ética empresarial y de la responsabilidad social corporativa. Veritas. Revista de Filosofía y Teología, 2(17), 205-224. https://www.reda lyc.org/articulo.oa?id=2911/291122924002

González, X. (17 de agosto de 2017). Las empresas que ayudan a cuidar a especies que están en vía de extinción. La Republica Recuperado de https://www.larepublica.co/responsabilidad-social/las-empresas-que-ayudan-a-cuida r-a-especies-que-estan-en-via-de-extincion-2537917 
Gürlek, M., \& Tuna, M. (2019). Corporate social responsibility and work engagement: Evidence from the hotel industry. Tourism Management Perspectives, 3, 195-208. https://doi.org/10.1016/j.tmp.2019.05.004

Gürlek, M., Düzgün, E., \& Uygur, S. (2017). How does corporate social responsibility create customer loyalty? The role of corporate image. Social Responsibility Journal, 13(3), 409-427. https://doi.org/10.1108/SRJ-10-2016-0177

Guthrie, J., \& Parker, L. D. (1989). Corporate Social Reporting: A Rebuttal of Legitimacy Theory. Accounting and Business Research, 19(76), 343-352. https://doi.org/10.1080/00014788.1989.9728863

Ham, C.-D., \& Kim, J. (2019). The effects of CSR communication in corporate crises: Examining the role of dispositional and situational CSR skepticism in context. Public Relations Review, (December 2018), 101792. h ttps://doi.org/10.1016/j.pubrev.2019.05.013

Havlová, K. (2015). What integrated reporting changed: The case study of early adopters. Procedia Economics and Finance, 34(15), 231-237. https://doi.org/10.1016/s2212-5671(15)01624-x

Hirtenstein, A. (2017). Grandes empresas petroleras se preocupan por reducir emisiones. Servicio Bloomberg Professional. Recuperado de https://www.bloomberg.com/latam/blog/grandes-empresas-petroleras-se-preocup an-por-reducir-emisiones/

Hogner, R. H. (1982). Corporate Social Reporting: Eight Decades of Development at US Steel. Research in Corporate performance and policy, 243-250.

Jensen, J. C., \& Berg, N. (2012). Determinants of traditional sustainability reporting versus integrated reporting, an institutionalist approach. Business Strategy and the Enviroment, 21(5), 299-316. https://doi.org/10.1002/bse. 740

Kansal, M., Joshi, M., \& Batra, G. (2014). Determinants of corporate social responsibility disclosures: Evidence from India. Advances in Accounting, 30(1), 217-229. https://doi.org/10.1016/j.adiac.2014.03.009

Lehman, C. (1983). Stalemate in Corporate Social Responsability Research. American Accounting Association Public Interest Section. Working paper. https://doi.org/10.1016/j.jclepro.2017.04.027

Manuel, A., Ramón, C., Teresa, E., Juan, F., Pablo, O., Joan, R., \& Juan, T. (2014). Métodos y técnicas cualitativas y cuantitativas aplicables a la investigación en ciencias sociales. Tirant humanidades México. 1.500-515. https://d oi.org/10.13140/RG.2.1.2083.9843

Maroun, W. (2017). Assuring the integrated report: Insights and recommendations from auditors and preparers. British Accounting Review, 49(3), 329-346. https://doi.org/10.1016/j.bar.2017.03.003

Medina, A. M., \& Alvarez, M. C. (2011). Balance en términos de responsabilidad social empresarial en las grandes empresas manufactureras del área Metropolitana del valle de Aburra. Revista visión contable, 9, 11-31. https:// doi.org/10.24142/rvc

Mesa, M. (2017). El ODS 16 sobre paz, seguridad y gobernanza: desafíos conceptuales, seguimiento y evaluación. Anuario CEIPAZ 2017-2018.10.29-62. https://dialnet.unirioja.es/servlet/articulo?codigo=6795699

Navarrete, O. J., \& Gallizo, L. J. (2018). Reporte Integrado como herramienta de transparencia empresarial. Revista Academia \& Negocios, 4(2), 71-82. https://dialnet.unirioja.es/servlet/articulo?codigo $=6750263$

Park, B. Il, \& Cave, A. H. (2018). Corporate social responsibility in international joint ventures: Empirical examinations in South Korea. International Business Review, 27(6), 1213-1228. https://doi.org/10.1016/j.ibu srev.2018.05.003

Pavlopoulos, A., Magnis, C., \& Iatridis, G. (2019). Integrated reporting: An accounting disclosure tool for high quality financial reporting. Research in International Business and Finance, 49(February), 13-40. https://doi.org/10.10 16/j.ribaf.2019.02.007

Preston, L \& Post, J. (1981). Private Management and Public Policy. California Management Review, 23(3), 56-62. h ttps://doi.org/10.2307/41172602

Price, J. M., \& Sun, W. (2017). Doing good and doing bad: The impact of corporate social responsibility and irresponsibility on firm performance. Journal of Business Research, 80(July), 82-97. https://doi.org/10.1016/j.j busres.2017.07.007 
Radley, Y. (2012). The value of extra-financial disclosure. What investors and analysts said. Global Reporting Initiative. Recuperado de https://www.globalreporting.org/information/news-and-press-center/Pages/Investors-and-an alysts-use-extra-financial-information-in-decision-making-suggests-new-research.aspx

Rayrnond, Q., \& Luc Van, C. (2005). Manual de la investigación en ciencias sociales. México D.F.: Limusa S.A.

Retolaza, J. L, San José L. \& Ruiz, M. (2010). Hacia un replanteamiento de la teoría de grupo de interés: Cuatro propuestas innovadoras. Recuperado de http://elib.bsu.by/handle/123456789/51768

Revista Dinero. (2019). Conozca los sectores que hacen más inversión social. Recuperado de https://www.dinero.co $\mathrm{m} /$ edicion-impresa/pais/articulo/conozca-los-sectores-que-hacen-mas-inversion-social/272632

Rivera, Y. A., Zorio A., \& García, M. A. (2016). El concepto de informe integrado como innovación en reporting corporativo. Journal of Innovation \& Knowledge, 1(3), 144-155. https://doi.org/10.1016/j.jik.2016.01.016

Robinson, S., \& Eilert, M. (2018). The role of message specificity in corporate social responsibility communication. Journal of Business Research, 90(July 2017), 260-268. https://doi.org/10.1016/j.jbusres.2018.05.028

Rodríguez, L. C., \& Ríos-Osorio, L. A. (2016). Evaluación de sostenibilidad con metodología GRI. Dimensión Empresarial, 14(2), 73-89. http://dx.doi.org/10.15665/rde.v14i2.659

Ross, M. (1992). Efficient Energy Use in Manufacturing. Proceedings of the National Academy of Sciences of the United States of America, 89(3), 827-831. Recuperado de https://www.jstor.org/stable/2358388

Rupley, K. H., Brown, D., \& Marshall, S. (2017). Evolution of corporate reporting: From stand-alone corporate social responsibility reporting to integrated reporting. Research in Accounting Regulation, 29(2), 172-176. https://do i.org/10.1016/j.racreg.2017.09.010

Sáez Martín, A., Haro de Rosario, A., \& Caba Pérez, M. D. (2014). Hacia una información corporativa integrada: evidencias en la industria de productos del cuidado de la salud. Revista Finanzas y Politica Económica, 6(2), 317-340. https://doi.org/10.14718/revfinanzpolitecon.2014.6.2.5

Sahin, M., \& Bayramoğlu, G. (2015). Decent Work in the Context of Corporate Social Responsibility. Journal of Advanced Management Science, (May 2016), 162-167. https://doi.org/10.12720/joams.3.2.162-167

Sanahuja, J. A., \& Tezanos, S. (2017). Del milenio a la sostenibilidad: retos y perspectivas de la Agenda 2030 para el desarrollo sostenible. Politica y Sociedad, 54(2), 521-543. https://doi.org/10.5209/POSO.51926

Sánchez, D., Rivera, J., \& Jaque, N. (2014). Capítulo 1 Divulgación de Información no Financiera en las Organizaciones: Una Mirada desde los Informes de Sostebilidad y los Reportes Integrados. La investigación e información corporativa e información corporativa. Recuperado de: https://investigacionuptag.wordpress.com /www.alinin.org

Schönborn, G., Berlin, C., Pinzone, M., Hanisch, C., Georgoulias, K., \& Lanz, M. (2019). Why social sustainability counts: The impact of corporate social sustainability culture on financial success. Sustainable Production and Consumption, 17, 1-10. https://doi.org/10.1016/j.spc.2018.08.008

Sharachchandra, L. (1991). Sustainable development: a critical review. World Development, 19(6), 607-621. https:// doi.org/10.1016/0305-750X(91)90197-P

Sìmnett, R., Vanstraelen, A., \& Chua, W. F. (2009). Assurance on sustainability reports: An international comparison. Accounting Review, 84(3), 937-967. https://doi.org/10.2308/accr.2009.84.3.937

Stewart, L. S. (2015). Growing demand for ESG information and standards: Understanding corporate opportunities as well as risks. Journal of Applied Corporate Finance, 27(2), 58-63. https://doi.org/10.1111/jacf.12118

Superintendencia de Sociedades. (2019). Comportamiento de las 1.000 empresas más grandes del sector real, por ingresos operacionales, Informe. 30. Recuperado de: https://www.supersociedades.gov.co/delegatura_aec/estu dios_financieros/Documents/SectorRealEconomia/Informe_las_1000_mas_grandes_sector_real_2018.pdf

Verrecchia, R. E. (1983). Discretionary disclosure. Journal of Accounting and Economics, 5, 179-194. https://doi.org /10.1016/0165-4101(83)90011-3 


\section{Notas}

* Artículo de investigación.

Reconocimientos Este trabajo es un resultado del proyecto de investigación "El Reporte Corporativo en Colombia y América Latina como mecanismo de rendición de cuentas", financiado por la Universidad de Antioquia (acta 2019-26130).

\section{Licencia Creative Commons CC BY 4.0}

Para citar este artículo: Villa-Barrientos, J. D., Escobar-Cruz, Y., \& Correa-Mejía, D. A. (2020). Evolución de la revelación de información no financiera: evidencia desde el contexto colombiano. Cuadernos de Contabilidad, 21. https://doi.org/10.11144/Javeriana.cc21.erif 